\title{
The number and distribution of esterase 6 alleles in populations of Drosophila melanogaster
}

\author{
J. Labate, ${ }^{*}$ \\ A. Bortoli, \\ A. Y. Game, \\ P. H. Cooke and \\ J. G. Oakeshott
}

\begin{abstract}
CSIRO Division of Entomology, GPO Box 1700 , Canberra, ACT 2601, Australia.
\end{abstract}

\begin{abstract}
High resolution electrophoretic analyses of the polymorphic esterase 6 enzyme have been carried out on 133 isoallelic lines from three Australian populations of Drosophila melanogaster spanning $25^{\circ}$ of latitude. These and previous data for 157 lines from another Australian population at an intermediate latitude reveal a total of 14 polymorphic esterase 6 allozymes, falling into five major mobility classes. Two classes, EST6-F and EST6-S, contain eleven of the allozymes but one allozyme, EST6-8 within the EST6-S class, is several times more common than any other. Variation in the frequency of this single allozyme can explain most of the latitudinal clines previously reported for the major EST6-F and EST6-S classes. Thermostability analyses of 52 of the Australian lines and 13 American lines reveal at least seven more EST6 variants within five of the allozymes, bringing the total number of variants to at least 21 . Of the six allozymes for which more than one line was subjected to thermostability analyses, only EST6-8 could not be partitioned into additional variants. This corroborates a previous finding that two different isolates of the Est6-8 allele have identical DNA sequences and suggests that this allele, although now the most common, has nevertheless arisen relatively recently.
\end{abstract}

\section{INTRODUCTION}

The finding of highly non-random patterns in the geographic distribution of gene frequencies has made the esterase 6 polymorphism of Drosophila melanogaster an important model for analysing the adaptive significance of electrophoretic variation. Esterase 6 is polymorphic for two major electrophoretic variants, EST6-F and EST6-S, and largescale latitudinal clines in their relative frequencies suggest that the two variants differ under natural selection (Oakeshott et al., 1981). This is supported by the finding of similar EST6-F and EST6-S variants showing similar latitudinal clines in the sibling species D. simulans (Anderson and Oakeshott, 1984).

However the interpretation of the clines is complicated by the finding of additional structural variation segregating within EST6-F and EST6-S. Cochrane and Richmond (1979) used in vitro thermostability criteria to resolve four variants

\footnotetext{
* Present address: Department of Ecology and Evolution, State University of New York at Stony Brook, Stony Brook, New York 11794, U.S.A.
}

within EST6-F and three within EST6-S in $D$ melanogaster. Subsequently, Cooke et al. (1987) used high resolution cellulose acetate electro. phoresis to detect a total of ten EST6 allozymes in this species. These allozymes fall into five major classes, EST6-U, EST6-VF, EST6-F', EST6-F and EST6-S, separated by relatively large electrophoretic mobility differences. The first three classes are uncommon and each contains only a single allozyme but the common EST6-F and EST6-S classes contain three and four minor mobility variants respectively. (Note that the terms "major" and "minor" are used throughout this paper to distinguish electrophoretic variants on the basis of large versus small differences in relative mobility; they do not refer to differences in their frequencies.)

What then is the relationship between the thermostability and minor mobility variants, and which of the additional variants underlie the latitudinal clines for the major EST6-F and EST6-S classes? To address these questions, we report here the frequencies of the minor mobility variants of EST6 in samples of isoallelic lines from three Australian populations of $D$. melanogaster covering 
$25^{\circ}$ of latitude. Fifty-two of these lines were then cross-classified for their EST6 thermostability phenotype.

\section{MATERIALS AND METHODS}

One hundred and thirty-three third chromosome isoallelic lines were extracted from populations collected at Darwin $\left(13^{\circ} \mathrm{S}, 131^{\circ} \mathrm{E}\right)$, Townsville $\left(19^{\circ} \mathrm{S}, 147^{\circ} \mathrm{E}\right)$ and Melbourne $\left(38^{\circ} \mathrm{S}, 145^{\circ} \mathrm{E}\right)$ during the summer of $1986 / 1987$. The extraction procedure was the same as that used by Cooke et al. (1987) to isolate 157 third chromosome isoallelic lines from a population at Coffs Harbour $\left(31^{\circ} \mathrm{S}, 153^{\circ} \mathrm{E}\right)$. Briefly, a standard backcrossing procedure was used to isolate individual third chromosomes from wild-caught males against a TM3 balancer chromosome (Lindsley and Grell, 1968). Homozygous lines were then established for those wild-caught third chromosomes that were viable and fertile as homozygotes. Otherwise heterozygotes were crossed to the $D f(3)$ vin $^{7}$ chromosome of Akam et al. (1978) which is deleted for the region (68C8-11 to 69B4-5) containing the Est6 locus (as 69A1). Hemizygous progeny were then used to establish pure-breeding lines for the Est 6 gene. Thus the homozygous and hemizygous isoallelic lines together represent a random sample of third chromosomes with respect to recessive viability or fertility genes.

Each isoallelic line was typed for its high resolution electrophoretic phenotype, using Titan III cellulose acetate plates (No. 3024 Helena Laboratories, Beaumont, Texas) and following the procedures of Cooke et al. (1987). The only modification to these procedures was that electrophoresis was carried out at room temperature for 110 minutes at $240 \mathrm{~V}$. As in Cooke et al. (1987), several homogenates from different cultures of each line were analysed and a phenotype was only assigned after repeated pairwise comparisons against all lines with similar or adjacent mobility phenotypes.

Thermostability analyses were carried out on 56 lines. Forty-four of these were randomly selected homozygous Darwin and Melbourne lines. Eight others were homozygous lines from Coffs Harbour isolated by Cooke et al. (1987); these were chosen as representatives of all six EST6 allozymes found among the homozygous lines from Coffs Harbour. The remaining four lines were control American lines of known EST6 thermostability phenotypes (Cochrane and Richmond 1979).
Three to five homogenates from different cultures of each of the above lines were assayed for thermostability. Homogenates were prepared from 4 day-old virgin males at a concentration of $17 \mathrm{mg}$ flies $/ 10 \mu \mathrm{l}$ buffer $(0 \cdot 1 \mathrm{M}$ phosphate $p \mathrm{H} \mathrm{6.8 \text {, }}$ $10 \mathrm{mg} / \mathrm{ml} \mathrm{BSA}$ ) following the procedures of Game and Oakeshott (1988). Replicate $30 \mu \mathrm{l}$ aliquots of each homogenate were heat-treated at $54^{\circ} \mathrm{C}$ for 35 minutes and then immediately placed on ice. EST6 activity was determined for these treated aliquots as well as for replicate $10 \mu l$ untreated aliquots of the same homogenates.

EST6 activity was measured by the end-point spectrophotometric assay of Sheehan et al. (1979) as modified by Game and Oakeshott (1989), except that the assay volume was scaled down to $210 \mu \mathrm{l}$ and the reactions were carried out in microtitre plates and read in a Titertek ${ }^{\circledR}$ Multiskan Elisa plate reader. The thermostability of EST6 in each homogenate was expressed as the angular transform of the ratio of the mean activity of the two replicate treated $30 \mu \mathrm{l}$ aliquots to three times the mean activity of the two replicate untreated $10 \mu \mathrm{l}$ aliquots.

\section{RESULTS}

Table 1 shows the relative mobilities and frequencis of the 14 EST6 allozymes identified among the Darwin, Townsville and Melbourne lines and the original Coffs Harbour sample of Cooke et al. (1987). Four of the allozymes had not been recorded in the Coffs Harbour sample and of these, two (EST6-4.5 and EST6-5.5, with relative mobilities of 1.065 and 1.05 , respectively) are minor mobility variants within the major EST6-F class and the other two (EST6-10 and EST6-11, relative mobilities 0.98 and 0.97 ) are minor variants within the major EST6-S class. This brings the total numbers of minor EST6-F and EST6-S variants to six and five respectively.

Two of the five minor EST6-F variants, EST6-3 and EST6-4.5, are uncommon or absent in all four samples. The frequencies of the other three vary substantially among populations and range from 0 to 25 per cent for EST6-4, from 0 to 42 per cent for EST6-5 and from 0 to 21 per cent for EST6-5.5. Each of these three allozymes is the most common EST6-F variant in at least one population.

In contrast only one of the six EST6-S variants exceeds a frequency of 20 per cent in any sample. This allozyme, EST6-8, is the most common EST6$\mathrm{S}$ variant in all four populations (35-66 per cent) 
Table 1 Relative electrophoretic mobilities and frequencies (per cent \pm S.E.) of the 14 EST6 allozymes found in four Australian population samples. Mobilities are expressed relative to that for EST6-8 which is defined as 1.00. The latitudes of the collection localities and the numbers of EST6 isoallelic lines scored are given in parentheses. Data for Coffs Harbour are taken from Cooke et al. (1987)

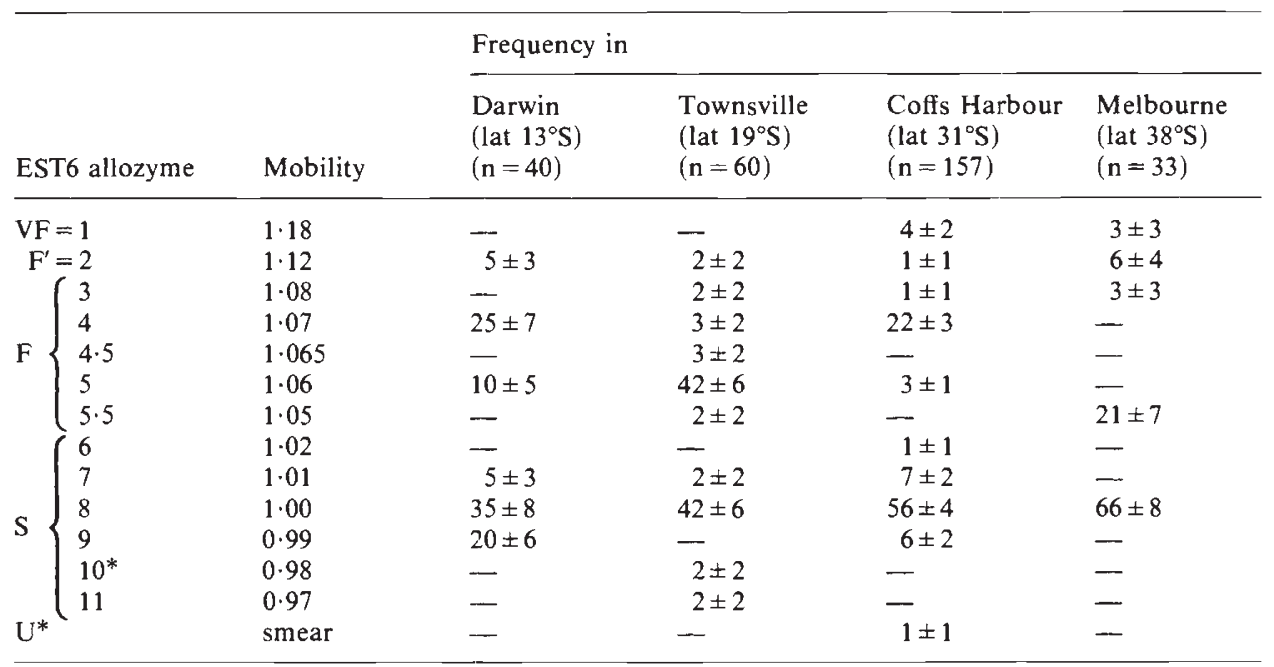

* Nomenclature for EST6-10 and EST6-U differs from that in Cooke et al. (1987). They did not find EST6-10 in their Coffs Harbour lines and they denoted EST6-U as EST6-10 instead.

and, on average, its frequency is at least ten times that of any of the other EST6-S allozymes.

Although the four populations sampled cover a $25^{\circ}$ latitudinal range, the small sizes of the samples from the latitudinal extremes (Darwin, 40 lines and Melbourne, 33 lines) make it difficult to assess clinal trends. Nevertheless the data are consistent with the clines for the major EST6-F and EST6-S classes reported by Oakeshott et al. (1981) and Anderson and Oakeshott (1984). The two northern populations, Darwin and Townsville, show higher frequencies of the major EST6-F allozyme class and lower frequencies of the major EST6-S allozyme class than do Coffs Harbour and Melbourne. Of the three more common minor variants within EST6-F, two, EST6-5 and to a lesser extent EST6-4, contribute to this difference; however the third, EST6-5.5, does not, being most common in the most southerly sample, from Melbourne. EST6-8, as the only common minor variant within EST6-S, shows the latitudinal trend very clearly, with a monotonic increase in frequency with increasing latitude from $35 \pm 8$ per cent in Darwin to $66 \pm 8$ per cent in Melbourne.

The EST6 enzyme activities of the untreated control aliquots (data not shown) for the thermostability analyses do not vary significantly among lines $\left(F_{(51,181)}=0.81, P>0.05\right)$. By comparison, the analyses of the original Coffs Harbour lines by Game and Oakeshott (1988) revealed highly significant activity differences among lines. The lack of significance of these differences in the present study may in part reflect the smaller number of lines sampled (fewer than half the replicates).

Fig. 1 shows the thermostabilities of the 56 lines assayed. The relative thermostabilities of the four American control lines concur with those reported by Cochrane and Richmond (1979), although the difference between the EST6-F1 and EST6-F2 lines (10 and 17 in fig. 1 ) is not statistically significant in this study.

There is highly significant variation in thermostability among the 52 Australian lines $\left(F_{(51,177)}=\right.$ $4.00, P<0.001)$ and much of this variation is among lines within minor mobility variants. Thus, there is significant heterogeneity among lines within five of the six minor variants for which more than one line was assayed. The five showing such heterogeneity are EST6-2, EST6-4, EST6-5, EST65.5 and EST6-9 (table 2). The one allozyme not showing heterogeneity among lines is EST6-8 $\left(F_{(26,53)}=0.73, P>0.05\right)$, even though many more lines were assayed for EST6-8 than for the other five allozymes. Compared to that within any of the 


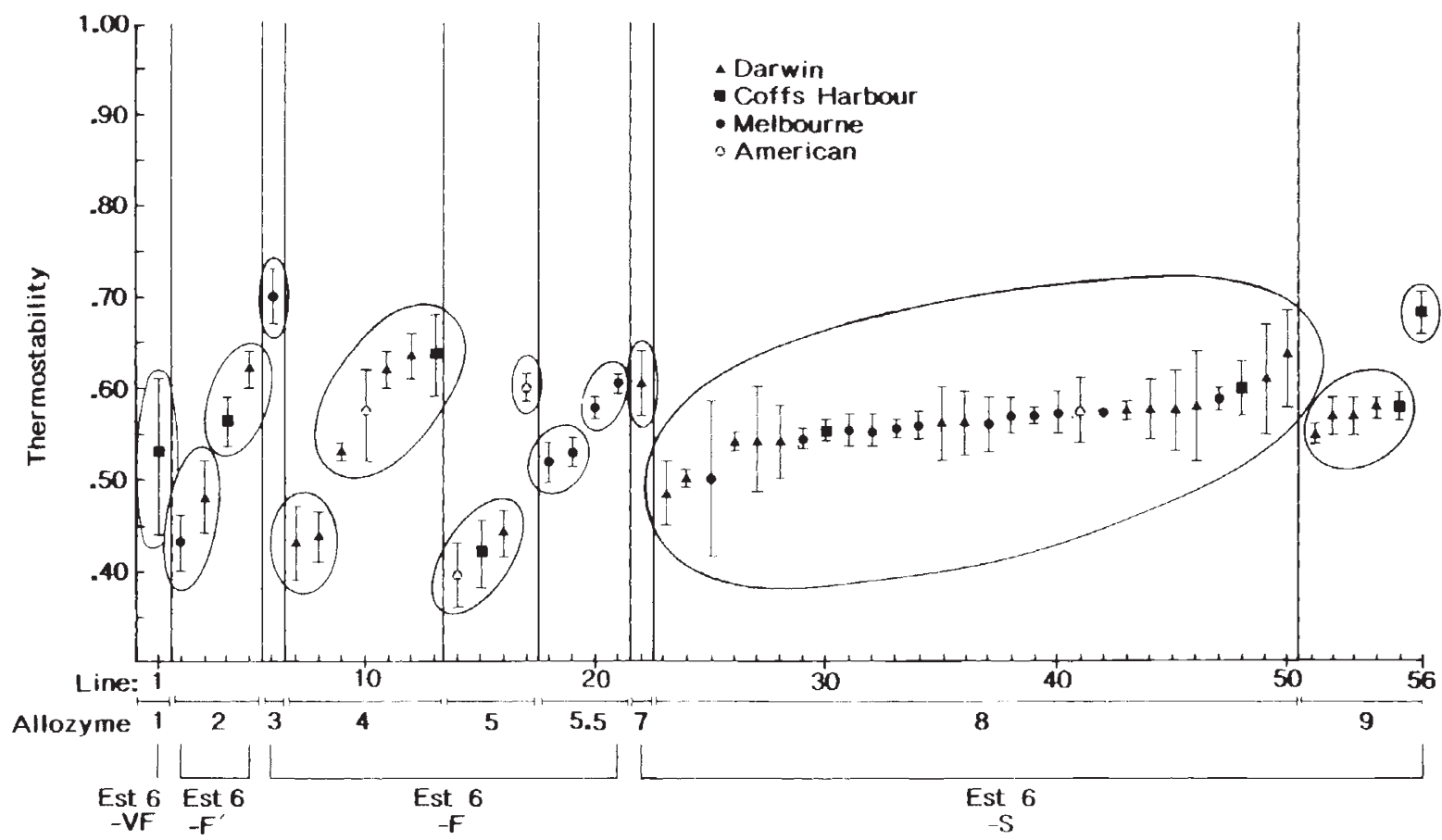

Figure 1 In vitro EST6 thermostabilities $(x \pm$ S.E.) of 56 isoallelic lines from four populations and covering nine EST6 allozymes. Thermostability is expressed as the angularly transformed proportion of activity remaining after 35 minutes at $54^{\circ} \mathrm{C}$. Lines within each allozyme with thermostabilities not significantly different from each other are encircled.

other five variants, the variance among lines within EST6-8 is considerably smaller (e.g. $F_{(6,26)}=$ $17 \cdot 22, P<0 \cdot 001$ compared to EST6-4).

Comparison with the control (line 41 in fig. 1) shows that EST6-8 corresponds to the thermostability variant EST6-S2 of Cochrane and Richmond (1979). This is consistent with the observa- tions that EST6-S2 was the most common thermostability variant in the four American populations scored by Cochrane and Richmond (1979), while EST6-8 is the most common allozyme in the four Australian populations.

Most of the heterogeneity among lines within the EST6-2, EST6-4, EST6-5, EST6-5.5 and EST6-

Table 2 Thermostability variation among lines within five allozymes. Lines within each allozyme are partitioned into low and high groups according to their thermostabilities. Means and standard errors of the thermostability values for each group are shown (with line numbers from fig. 1 in parentheses underneath), together with the percentage of the variation among lines explained by the two groups

\begin{tabular}{|c|c|c|c|c|}
\hline Allozyme & $\begin{array}{l}\text { Variation } \\
\text { among linest }\end{array}$ & Low group & High group & $\begin{array}{l}\text { Per cent variation } \\
\text { due to groups }\end{array}$ \\
\hline EST6-2 & $F(3,24)=8 \cdot 02^{* * *}$ & $\begin{array}{c}0 \cdot 45+0 \cdot 03 \\
(2,3)\end{array}$ & $\begin{array}{l}0 \cdot 59 \pm 0 \cdot 02 \\
(4,5)\end{array}$ & 87 \\
\hline EST6-4 & $F(6,41)=5 \cdot 92^{* * *}$ & $\begin{array}{l}0 \cdot 43 \pm 0 \cdot 02 \\
(7,8)\end{array}$ & $\begin{array}{l}0.59 \pm 0.02 \\
(9-13)\end{array}$ & 78 \\
\hline EST6-5 & $F(3,22)=9 \cdot 67^{* * *}$ & $\begin{array}{l}0.42 \pm 0.02 \\
(14-16)\end{array}$ & $\begin{array}{l}0.60 \pm 0.02 \\
(17)\end{array}$ & 97 \\
\hline EST6-5.5 & $F(3,12)=6 \cdot 22^{* * *}$ & $\begin{array}{l}0.52 \pm 0.01 \\
(18,19)\end{array}$ & $\begin{array}{l}0 \cdot 59+0 \cdot 01 \\
(20,21)\end{array}$ & 95 \\
\hline EST6-9 & $F(5,34)=8 \cdot 89^{* * *}$ & $\begin{array}{l}0.57 \pm 0.01 \\
(51-55)\end{array}$ & $\begin{array}{l}0.68 \pm 0.02 \\
(56)\end{array}$ & 96 \\
\hline
\end{tabular}

† Includes control lines.

$* * * P<0.001$. 
9 allozymes can be explained by partitioning the lines in each of these five allozymes into two groups (table 2). Even the most parsimonious explanation thus adds a further five to the number of EST6 variants, taking it from 14 to 19 .

Results from the similar but smaller data set of Cooke et al. (1987) show that the total number of EST6 variants is even higher. They carried out high resolution electrophoretic analysis on 13 isoallelic lines surviving from the thermostability study of Cochrane and Richmond (1979). (Four of these lines are the controls in the present work.) Only three of these lines bore EST6-S thermostability variants, with one bearing EST6-S1 electrophoresing as EST6-9 and the other two, bearing EST6-S2, electrophoresing as EST6-8 (further supporting the correspondence between EST6-S2 and EST6-8 proposed above). However the other ten lines included all four EST6-F thermostability variants, with four lines bearing EST6-F1, EST6-F2 and EST6-F3 all electrophoresing as EST6-4 and the other six lines, bearing EST6-F1, EST6-F3 and EST6-F4, all electrophoresing as EST6-5. Allozymes EST6-4 and EST6-5 hence each contain at least three thermostability variants and these, together with the present data, take the total number of EST6 variants identified to 21 .

\section{DISCUSSION}

Fourteen EST6 allozymes have been identified by high resolution cellulose acetate electrophoresis of 290 isoallelic lines from four Australian populations of $D$. melanogaster. Thermostability analyses of 52 of these lines and another 13 American lines reveals the presence of at least another seven variants within these EST6 allozymes, taking the total number of EST6 variants to at least 21. Previous genetic analyses show that both classes of variant map to the Est 6 locus (Cochrane and Richmond 1979, Cooke et al. 1987), and it is likely that the additional variation now identified also does so. There are probably many more Est6 alleles still undescribed, since only six of the 14 allozymes were scored for thermostability in more than one line.

Seventeen of the 21 EST6 variants so far resolved lie within the common EST6-F and EST6-S electrophoretic classes, ten within EST6-F and seven within EST6-S. One variant within EST6-S, defined electrophoretically as EST6-8 and by its thermostability as EST6-S2, is particularly common (35-66 per cent frequency in the four Aus- tralian populations and 51-58 per cent in four American populations; table 1 and Cochrane and Richmond 1979). However, the other EST6-F and EST6-S variants are each uncommon and even the three most frequent EST6-F allozymes can each be subdivided into two or three thermostability variants. The polymorphism therefore comprises a single common allele and many relatively uncommon variants.

As the only common variant, EST6-8 makes a large contribution to the latitudinal clines for the major EST6-F and EST6-S mobility classes. It shows a monotonic rise in frequency from 35 to 66 per cent over the four Australian populations and $25^{\circ}$ of latitude sampled. However EST6- 8 may not be the only minor mobility variant which contributes to the clines for the major EST6-F and EST6-S allozyme classes. The power of these analyses to detect latitudinal trends for less common variants is limited by the small numbers of populations and lines scored. Two of the minor variants within the EST6-F class, namely EST6-4 and EST6-5, do show north/south differences which are at least consistent with the overall EST6$F$ cline. However, even the interpretation of these differences is complicated by the fact that both the EST6-4 and EST6-5 alloaymes each comprise at least three thermostability variants.

While it is thus not clear whether EST6-4 and EST6-5 contribute to the clines for the major EST6$F$ and EST6-5 classes, it is clear that some of the other variants do not. In particular, EST6-F variant EST6-5.5 is only common ( $21 \pm 7$ per cent) in the most southerly population (Melbourne), whereas in the overall latitudinal cline EST6-F is less common at higher latitudes. Hence the latitudinal selection generating the major EST6-F and EST6-S clines does not operate uniformly on all minor variants within the two major allozyme classes.

Such heterogeneity among the minor variants may also contribute to some discrepancies between previous studies on biochemical and fitness differences between EST6-F and EST6-S genotypes. For example, Danford and Beardmore (1980) found that purified EST6-S enzyme was more thermostable than purified EST6-F, whereas White et al. (1988) reported the opposite. Similar discrepancies exist among studies which compare the effects of high temperatures on the fitnesses of Est6-F and Est6-S genotypes (e.g., Aslund and Rasmuson 1967, Minawa and Birley 1978, Oakeshott 1979, Abedin et al. 1980). Perhaps the different studies utilised different minor mobility and thermostability variants, at least within the major EST6-F class. 
The common variant, EST6-8 (= EST6-S2) is the only EST6 variant identified by electrophoresis which cannot yet be partitioned into additional variants by thermostability criteria. This is consistent with the findings of Cooke and Oakeshott (1988) that two alleles encoding EST6-8 isolated from populations on different continents have identical DNA sequences, whereas separate isolates of the alleles encoding the EST6-4 and EST6-9 allozymes differ at 13 and six sites respectively (including three each which cause amino acid replacements). Our results thus support their conclusion that the Est6-8 allele has arisen relatively recently and our finding that this allele is now about ten times more common than any other variant further suggests a recent rapid rise in its frequency.

It is relevant in this context that EST6-8 frequency is higher at higher latitudes in Australia. If the same is true elsewhere (which seems likely given the similar directions of the overall EST6-S clines across continents), then the latitudinal clines probably do not simply represent a transient stage in the spread of EST6-8 away from an equatorial origin. It follows that the selective process underlying its spatial distribution is not the same as the process which has led to its recent increase in frequency. The latter process may be a selective one or may be random drift.

Acknowledgements We are grateful to Julia Davidson and Wayne Knibb for collecting the three Drosophila populations studied here. We also thank Peter Christian, Joanne Daly, Mira Dumancic, Marion Healy, Jill Karotam, Mark Myers, Robyn Russell, Rollin Richmond, Marlene Saad and Lis van Papenrecht for valuable advice and discussions. In particular we thank Mira Dumancic and Marion Healy for modifying the EST6 activity assay for use with limited material.

\section{REFERENCES}

ABEDIN, K., McNAMARA, S., OSTERBUR, D. AND STEINER, W. W. M. 1980. Studies on the effect of temperature on fitness and fecundity at the esterase 6 locus in $D$. melanogaster. Drosophila Inform. Serv., 55, 10-11.
AKAM, M. E., ROBERTS, D. B., RICHARDS, G. P. AND ASHBURNER, M. 1978. Drosophila: the genetics of two major larval proteins. Cell, 13, 215-225.

ANDERSON, P. R. AND OAKESHOTT, J. G. 1984. Parallel geographic patterns of allozyme variation in two sibling Drosophila species. Nature, 308, 729-731.

ASLUND, S. E. AND RASMUSON, M. 1976. Mating behaviour as a fitness component in maintaining allozyme polymorphism in Drosophila melanogaster. Hereditas, 82, 175 178.

COChrane, B. J. And RIChmond, R. C. 1979. Studies of esterase 6 in Drosophila melanogaster. II. The genetics and frequency distributions of naturally occurring variants studied by electrophoretic and heat stability criteria. Genetics, 93, 461-478.

COOKE, P. H. AND OAKESHOTT, J. G. 1988. Amino acid polymorphisms for esterase 6 in Drosophila melanogaster. Proc. Natl Acad. Sci. USA (In press).

COOKE, P. H., RICHMOND, R. C. AND OAKESHOTT, J. G. 1987 High resolution electrophoretic variation at the esterase 6 locus in a natural population of Drosophila melanogaster. Heredity, 59, 259-264.

DANFORD, N. D., AND BEARDMORE, J. A. 1979. Biochemical properties of esterase 6 in Drosophila melanogaster. Biochem. Genet., 17, 1-22.

GAME, A. Y. AND OAKESHOTT, J. G. 1989. Variation in the activity and amount of esterase 6 in a natural population of Drosophila melanogaster. Heredity 62, 27-34.

LINDSLEY, D. L. AND GRELL, E. H. 1968. Genetic Variations of Drosophila melanogaster. Carnegie Institute, Washington. 472pp.

MINAWA, A. AND BIRLEY, A. J. 1978. The genetical response to natural selection by varied environments. I. Short-term observations. Heredity, 40, 39-50.

OAKESHOTT, J. G. 1979. Selection affecting enzyme polymorphisms in natural populations of Drosophila melanogaster. Oecologia, 43, 341-354.

OAKESHOTT, J. G., CHAMBERS, G. K., GIBSON, J. B. AND WILLCOCKS, D. A. 1981. Latitudinal relationships of esterase 6 and phosphoglucomutase gene frequencies in Drosophila melanogaster. Heredity, 47, 385-396.

SHEEHAN, K., RICHMOND, R. C. AND COCHRANE, B. J. 1979. Studies of esterase 6 in Drosophila melanogaster. III. The developmental pattern and tissue distribution. Insect Biochem., 9, 443-450.

WHITE, M. M., MANE, S. D. AND RICHMOND, R. C. 1988. Studies of esterase 6 in Drosophila melanogaster. XVIII. Biochemical differences between the slow and fast allozymes. Mol. Biol. Evol, , 5, 41-62. 